\title{
Modeling and Analysis of Local Comprehensive Minutia Relation for Fingerprint Matching
}

\author{
Xiaoguang He, Jie Tian, Senior Member, IEEE, Liang Li, Yuliang He, and Xin Yang
}

\begin{abstract}
This paper introduces a robust fingerprint matching scheme based on the comprehensive minutia and the binary relation between minutiae. In the method, a fingerprint is represented as a graph, of which the comprehensive minutiae act as the vertex set and the local binary minutia relations provide the edge set. Then, the transformation-invariant and transformationvariant features are extracted from the binary relation. The transformation-invariant features are suitable to estimate the local matching probability, whereas the transformation-variant features are used to model the fingerprint rotation transformation with the adaptive Parzen window. Finally, the fingerprint matching is conducted with the variable bounded box method and iterative strategy. The experiments demonstrate that the proposed scheme is effective and robust in fingerprint alignment and matching.
\end{abstract}

Index Terms-Adaptive Parzen window, binary minutia relation, fingerprint identification, transformation-invariant feature, transformation-variant feature.

\section{INTRODUCTION}

A T PRESENT, fingerprint identification is much more reliable than most other biometric identification methods such as signature, face, and speech [1]. Various algorithms and techniques have been developed rapidly for fingerprint identification systems in the past decade. In fact, a fingerprint is the identity card that people carry for a lifetime. The classical fingerprint identification was applied in security systems like prison and criminal identification [1]. Recently, with the development of the technology, it is increasingly used for civilian daily life, such as access control, financial security, verification of firearm purchaser [2], etc.

Manuscript received April 29, 2006; revised October 10, 2006. This work was supported in part by the Project of the National Science Foundation for Distinguished Young Scholars of China under Grant 60225008, by the Key Project of the National Natural Science Foundation of China under Grants 60332010 and 60575007, by the Project for Young Scientists' Foundation of the National Natural Science of China under Grant 60303022, by the Project of the Natural Science Foundation of Beijing under Grant 4052026, by Chair Professors of the Cheung Kong Scholars Program, by PCSIRT, and by the Chinese Academy of Sciences Hundred Talents Program. This paper was recommended by Guest Editor N. Ratha.

$\mathrm{X}$. He, L. Li, Y. He, and X. Yang are with the Key Laboratory of Complex Systems and Intelligence Science, Institute of Automation, Chinese Academy of Sciences, Beijing 100080, China (e-mail: xiaoguang.he@ia.ac.cn; Liang.Li@ia.ac.cn; yuliang.he@ia.ac.cn; xin.yang@ia.ac.cn).

J. Tian is with the Key Laboratory of Complex Systems and Intelligence Science, Institute of Automation, Chinese Academy of Sciences, Beijing 100080, China, and also with the Life Science Center, Xidian University, Xi' an 710071, China (e-mail: tian@ieee.org).

Color versions of one or more of the figures in this paper are available online at http://ieeexplore.iee.org.

Digital Object Identifier 10.1109/TSMCB.2006.890285
A fingerprint is a pattern of ridges and valleys on skin surface. The uniqueness of a fingerprint can be determined with an overall pattern of ridges and valleys as well as local ridge anomalies, such as ridge endings and bifurcations, i.e., minutiae. Many experts have designed fingerprint representation schemes under the strong assumption that the input fingerprint and template fingerprint are acquired by the same sensor. Therefore, those schemes usually characterize the same intensity range and admit a certain type of noise. In current years, techniques [3]-[8] are developed to recover geometric distortion and misalignment of fingerprints. However, these methods are time and memory consuming since they usually align all minutiae one by one with the local transformation information.

In this paper, a robust fingerprint matching scheme is designed to explore comprehensive information of minutiae and ridges and the relations between minutiae. The method introduces a graph in fingerprint representation. In the graph, the vertex set is the comprehensive minutiae, and the edge set is the local binary minutia structures. Local structure is subject to the positional constraints, and it helps to represent a local fingerprint region and prevent false matching caused by insufficient minutiae. Compared with the ternary minutia structure as other researchers adopted [3]-[8], [19], binary structure makes a proper tradeoff between the performance and computational expense. The proposed feature representation is inexpensive in time and memory cost.

Two types of features are extracted from the binary comprehensive minutia structure. One is the transformation-invariant features, which are used for the local matching probability measurement between local structures. Another is the transformation-variant features, and they are used to model the rotation transformation with the adaptive Parzen window, which statistically explores the transformation information from local structures and admits the periodic property of rotation angle. Finally, the variable bounded box method [9] and iterative strategy are used for rechecking local matching probability. Both the variable bounded box method and iterative strategy globally reduce the influence of deformation on matching. Experimental results on the database of the International Fingerprint Verification Competition (FVC) 2002 [17] have proven that our technique is efficient in terms of fingerprint alignment and matching.

The rest of this paper is organized as follows: Section II introduces and analyses the representation of fingerprint feature. Section III describes the measurement of transformation parameter. Section IV presents the scheme of fingerprint matching. Section V provides the experimental results. The final section concludes our work with future perspectives. 


\section{REPRESENTATION OF Fingerprint FEATURE}

Many popular fingerprint representation schemes, based on image analysis, can be classified into three types. The first type is the minutia-based technologies, which are predominantly dependent on local landmarks [1], [3], [10], i.e., ridge endings and bifurcates. This is widely used for its time saving and less memory consumption. However, a minutia set cannot characterize overall patterns of a fingerprint, and it is hard to further improve the performance. The second type is the exclusive global feature-based approaches [11]. With these methods, the holistic patterns of fingerprint texture are used to calculate the maximum mutual information between two fingerprints. The approaches are employed not only in identification [11], [12] but also in indexing [13], [14]. However, these methods require the exact determination of central point, and it is difficult to deal with distortion in the fingerprints. Moreover, the exclusive global information-based method needs much memory to store a fingerprint template. The third type is technologies based on comprehensive feature [12], [15]. In these schemes, fingerprints are matched by fusing minutiae, local features, and global features with a hybrid method. Local features accelerate the alignment of the input minutia patterns in different sizes, and the global features are used to overcome the shortage of minutiae and local features in lowquality fingerprints. In addition, these methods may combine various technologies, such as bounding box method and mutual information method. These approaches are popular for their robust performance with acceptable memory expense in recent years. However, these methods are not omnipotent for some special conditions with large deformation.

This paper introduces a comprehensive feature-based technique with two novel aspects: 1) a fingerprint pattern is characterized by the comprehensive minutiae and the binary relations between minutiae; and 2) as an improvement of our previous work [9] in alignment, the adaptive Parzen window is proposed to model the relationship between the local matching probability and the fingerprint transformation.

\section{A. Comprehensive Minutia}

A poor-quality fingerprint may be too dry or too wet, or the foreground area may be narrow with insufficient reliable minutiae. In some cases, even two fingerprints from the same finger fail to match for lack of common minutiae. Therefore, associated ridge information is combined to improve the fingerprint representation. As demonstrated in Fig. 1, a comprehensive minutia $M_{n}$ includes a minutia and the associated ridge feature, formally

$$
M_{n}=\left\{x_{n}, y_{n}, \theta_{n}, \beta_{n}\right\} \cup\left\{\varphi_{n m} \mid m=1,2, \ldots, L\right\}
$$

where $\left(x_{n}, y_{n}\right)$ is the coordinate, and $\theta_{n}$ is the tangent direction. $\beta_{n}$ is the local gray variance of an area centered on $\left(x_{n}, y_{n}\right)$. $\varphi_{n m}$ is the direction from $\left(x_{n}, y_{n}\right)$ to $R_{n m}$, which is a point sampled on the ridge derived from the minutia. $\left\{\varphi_{n m} \mid m=\right.$ $1,2, \ldots, L\}$ embodies the information of the ridge curvature and the local shape, and $L$ is the number of sampled points on the associated ridge. Here, the type information of the minutiae,

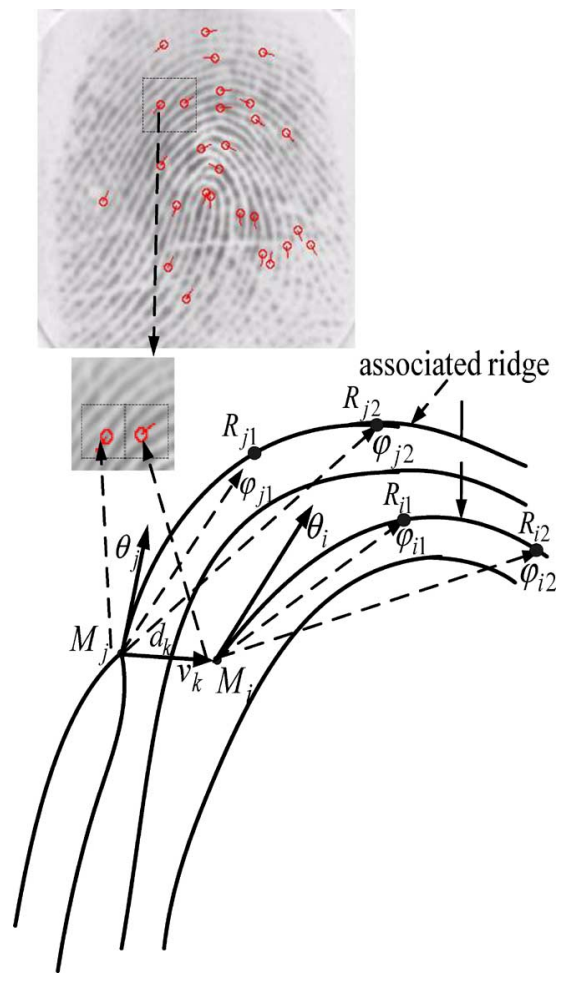

Fig. 1. Comprehensive minutiae and binary comprehensive minutia structure.

e.g., ending or bifurcate, is not employed since it usually makes false matching in our experiments.

In this paper, the comprehensive minutia set of a fingerprint $F$ is denoted as $M^{F}=\left\{M_{n} \mid n=1,2, \ldots, m(F)\right\}$, where $m(F)$ is the minutia number of $F . M^{F}$ contains the comprehensive information of all minutiae.

\section{B. Binary Comprehensive Minutia Structure}

In fingerprint representation, minutia is a unary property. There is the binary or higher order relation that conveys the contextual constraints, which are crucial in fingerprint matching. In this algorithm, binary structure between minutiae is introduced. For each pair of comprehensive minutia points $M_{i}$ and $M_{j}$, where $M_{i}, M_{j} \in M^{F}$ and $M_{i} \neq M_{j}$, if their Euclidean distance $d\left(M_{i}, M_{j}\right)=\sqrt{\left(x_{i}-x_{j}\right)^{2}+\left(y_{i}-y_{j}\right)^{2}}$ satisfies $d l \leq d\left(M_{i}, M_{j}\right) \leq d h$, then $M_{i}$ and $M_{j}$ are connected as a local binary structure $E_{k}$, formally

$$
E_{k}=\left\{s_{k}, e_{k}\right\} \cup\left\{v_{k}, \theta_{k}^{s}, \theta_{k}^{e}, \Psi_{k}^{s}, \Psi_{k}^{e}\right\} \cup\left\{d_{k}, \beta_{k}^{s}, \beta_{k}^{e}, \alpha_{k}^{s}, \alpha_{k}^{e}\right\}
$$

where $s_{k}$ and $e_{k}$ denote the serial numbers of the binary minutiae in minutia set $M^{F}$. As shown in Fig. $1, s_{k}=i$ and $e_{k}=j$. $\left\{v_{k}, \theta_{k}^{s}, \theta_{k}^{e}, \Psi_{k}^{s}, \Psi_{k}^{e}\right\}$ stands for the transformation-variant features; it describes the information that is affected by fingerprint transformation, where $v_{k}=\arctan \left(\left(y_{i}-y_{j}\right) /\left(x_{i}-\right.\right.$ $\left.\left.x_{j}\right)\right), \theta_{k}^{s}=\theta_{i}, \theta_{k}^{e}=\theta_{j}, \Psi_{k}^{s}=\left\{\varphi_{i m} \mid m=1,2, \ldots, L\right\}$, and $\Psi_{k}^{e}=\left\{\varphi_{j m} \mid m=1,2, \ldots, L\right\} . \quad\left\{d_{k}, \beta_{k}^{s}, \beta_{k}^{e}, \alpha_{k}^{s}, \alpha_{k}^{e}\right\} \quad$ is the transformation-invariant features; it represents the unchanged information under transformation, where $d_{k}=d\left(M_{i}, M_{j}\right)$, $\beta_{k}^{s}=\beta_{i}, \beta_{k}^{e}=\beta_{j}, \alpha_{k}^{s}=\theta_{i}-v_{k}$, and $\alpha_{k}^{e}=\theta_{j}-v_{k}$. 
The binary comprehensive minutia structure set is formally $E^{F}=\left\{E_{k} \mid k=1,2, \ldots, e(F)\right\}$, where $e(F)$ is the number of structures. $e(F)$ is much smaller than $m(F)(m(F)-1) / 2$ because the Euclidean distance $d$ of most minutia pair do not satisfy $d l \leq d \leq d h$. The size of $E^{F}$ can be controlled by modifying the values of $d l$ and $d h$. The minutia set $M^{F}$ and the binary structure set $E^{F}$ represent fingerprint $F$ as a graph $G^{F}=\left(M^{F}, E^{F}\right)$, where $M^{F}$ acts as the vertex set, and $E^{F}$ provides the edge set. $G^{F}$ explores the first- and second-order minutia relations of fingerprint $F$, and the higher order relations behave as the connected subgraphs of $G^{F}$.

\section{Measurement of Transformation Parameter}

It is important to align the input minutiae with the template during matching. The alignment generally includes rotation, translation, and shearing. This matching algorithm is designed assuming that the input and template fingerprints are captured by the same device in the same condition but with little scaling deformation. Since the fingerprint matching performs well in polar coordinate, the translation of the input features to the template is not concerned if the central point is set in advance. One of the most important tasks in alignment is to find the optimal rotation parameter.

\section{A. Matching Probability of Binary Comprehensive Minutia Structure}

Since the transformation-invariant features remain unchanged under fingerprint transformation, they are ideal for the matching probability estimation of local comprehensive minutia structures. For each pair of local structures $E_{i}$ and $E_{t}$, where $E_{i} \in E^{I}, E_{t} \in E^{T}$, and $E^{I}$ and $E^{T}$ denote the local structure sets of input fingerprint $I$ and template fingerprint $T$, respectively, then similarity $S_{i t}$ between $E_{i}$ and $E_{t}$ is estimated, formally

$$
\begin{aligned}
S_{i t}= & \left\{\begin{array}{cc}
0, & e_{i t}>\varepsilon \text { and } e_{i t}^{\prime}>\varepsilon \\
1-\frac{\min \left(e_{i t}, e_{i t}^{\prime}\right)}{\varepsilon}, & \text { otherwise }
\end{array}\right. \\
e_{i t}= & \left(d_{i}-d_{t}\right)^{2}+\left(\beta_{i}^{s}-\beta_{t}^{s}\right)^{2}+\left(\beta_{i}^{e}-\beta_{t}^{e}\right)^{2} \\
& \left.\quad+\left(\alpha_{i}^{s}-\alpha_{t}^{s}\right)^{2}+\left(\alpha_{i}^{e}-\alpha_{t}^{e}\right)^{2}\right)^{\frac{1}{2}} \\
e_{i t}^{\prime}= & \left(\left(d_{i}-d_{t}\right)^{2}+\left(\beta_{i}^{s}-\beta_{t}^{e}\right)^{2}+\left(\beta_{i}^{e}-\beta_{t}^{s}\right)^{2}\right. \\
& \left.\quad+\left(\alpha_{i}^{s}-\alpha_{t}^{e}-180\right)^{2}+\left(\alpha_{i}^{e}-\alpha_{t}^{s}-180\right)^{2}\right)^{\frac{1}{2}}
\end{aligned}
$$

where $\varepsilon$ is the matching threshold of transformation-invariant feature. If $S_{i t}=0, M_{i}$ and $M_{t}$ are not matched; otherwise they are. The more similar the $E_{i}$ and $E_{t}$ are, the larger the $S_{i t}$ is. However, two local structures, which are not from the same location of the same fingerprint, can be false matched accidentally. The false-matching cases can be excluded with the variable bounded box method, and the detail is presented in Section IV. All of the matching probability values construct a similarity matrix $S=\left[S_{i t}\right]_{1 \leq i \leq e(I), 1 \leq t \leq e(T)}$, which represents the local comprehensive similarity between fingerprints $I$ and $T$. In the next section, the rotation parameter is statistically analyzed with $S$, and in Section IV, $S$ is used for adjusting translation transformation with iterative strategy.

\section{B. Adaptive Parzen Window for Modeling Rotation Transformation}

The transformation-variant feature is useful for the rotation parameter estimation since it reflects the rotation transformation of a fingerprint. The local rotation parameter between local structures $E_{i}$ and $E_{t}$ is denoted as $\delta_{i t}$, and it is estimated as follows:

$$
\begin{aligned}
\delta_{i t}= & \frac{\Delta v_{i t}+\Delta \theta_{i t}+\Delta \Psi_{i t}}{3} \\
\Delta v_{i t}= & v_{i}-v_{t} \\
\Delta \theta_{i t}= & \frac{\theta_{i}^{s}+\theta_{i}^{e}-\theta_{t}^{s}-\theta_{t}^{e}}{2} \\
\Delta \Psi_{i t}=\frac{1}{2 L}\left(\varphi_{s_{i} 1}+\varphi_{s_{i} 2}+\cdots+\varphi_{s_{i} L}\right) & \\
& +\left(\varphi_{e_{i} 1}+\varphi_{e_{i} 2}+\cdots+\varphi_{e_{i} L}\right) \\
& -\left(\varphi_{s_{t} 1}+\varphi_{s_{t} 2}+\cdots+\varphi_{s_{t} L}\right) \\
& \left.-\left(\varphi_{e_{t} 1}+\varphi_{e_{t} 2}+\cdots+\varphi_{e_{t} L}\right)\right) .
\end{aligned}
$$

Then, the Parzen window is an effective method for estimating the probability density. When Gaussian function is chosen as the smooth kernel, the probability density $f(\delta)$ of rotation parameters $\delta$ is formally

$$
\begin{aligned}
f(\delta) & =\frac{\sum_{1 \leq i \leq e(I)} \sum_{1 \leq t \leq e(T)} K\left(\delta-\delta_{i t}\right)}{e(I) \cdot e(T)} \\
K\left(\delta-\delta_{i t}\right) & =\frac{1}{\sqrt{2 \pi \sigma^{2}}} \exp \left(-\frac{\left(\delta-\delta_{i t}\right)^{2}}{2 \sigma^{2}}\right)
\end{aligned}
$$

where $\sigma$ controls the size of the Parzen window. However, the Parzen window is not appropriate for the estimation since $f(\delta)$ is a periodic function and the similarity information of local binary structures is very important for the estimation. Therefore, the adaptive Parzen window is proposed as follows:

$$
\begin{aligned}
f(\delta) & =\frac{\sum_{1 \leq i \leq e(I)} \sum_{1 \leq t \leq e(T)} K\left(\delta-\delta_{i t}\right)}{e(I) \cdot e(T)} \\
K\left(\delta-\delta_{i t}\right) & =\left\{\begin{array}{cl}
\sum_{n=-\infty}^{+\infty} \frac{1}{\sqrt{2 \pi \sigma_{i t}^{2}}} \exp \left(-\frac{\left(\delta-\delta_{i t}+360 n\right)^{2}}{2 \sigma_{i t}^{2}}\right), & S_{i t} \neq 0 \\
\frac{1}{360}, & S_{i t}=0
\end{array}\right.
\end{aligned}
$$

where $\sigma_{i t}^{2}=1 / 2 \pi\left(a S_{i t}\right)^{2}$, and $a$ is an experiential value. Compared with the Parzen window, the window size of every sample's smooth kernel is flexible, and it is determined by the corresponding similarity. Given a rotation angle $\delta_{i t}$ of a local structure, the probability density function conditioned 
on $\delta_{i t}$ is formally $f\left(\delta \mid \delta_{i t}\right)=\exp \left(-\left(\delta-\delta_{i t}\right)^{2} / 2 \sigma_{i t}^{2}\right) / \sqrt{2 \pi \sigma_{i t}^{2}}$. The more similar the local structures are, the more crucial the corresponding rotation angle estimation is. When it is assumed that the probabilistic certainty is in proportion to the similarity, i.e., $\left.f\left(\delta \mid \delta_{i t}\right)\right|_{\delta=\delta_{i t}}=1 / \sqrt{2 \pi \sigma_{i t}^{2}}=a \cdot S_{i t}$, then $\sigma_{i t}^{2}=$ $1 /\left(2 \pi\left(a S_{i t}\right)^{2}\right)$. The larger the $S_{i t}$ is, the sharper the $f\left(\delta \mid \delta_{i t}\right)$ is. In other words, the larger the similarity of two local structures is, the more definite the fingerprint rotation angle equals to $\delta_{i t}$. When two local structures are not matched, i.e., $S_{i t}=0$, the corresponding angle $\delta_{i t}$ cannot make any contribution to the final estimation. In this case, $f\left(\delta \mid \delta_{i t}\right)=1 / 360$. In (13), it does not need to sum the periodic responses from $-\infty$ to $+\infty$; the sum from -3 to +3 can yield a satisfying approach.

To accurately calculate the transformation parameters, a confidence interval $\left[\delta_{\max }-\sigma_{\delta}, \delta_{\max }+\sigma_{\delta}\right]$ is defined, where $\delta_{\max }$ satisfies $f\left(\delta_{\max }\right)=\max _{\delta}\{f(\delta)\}$, and $\sigma_{\delta}$ satisfies $\sigma_{\delta}^{2}=\sum_{i t}\left(S_{i t} \cdot\left(\delta_{i t}-\bar{\delta}\right)^{2}\right) / \sum_{i t} S_{i t}$, where $\bar{\delta}=\sum_{i t}\left(S_{i t}\right.$. $\left.\delta_{i t}\right) / \sum_{i t} S_{i t}$. The confidence interval can reduce the effect of false matching of local binary structures because the falsematching contribution to $f(\delta)$ mainly concentrates on the outside of the confidence interval. Moreover, as denoted in (14), it is effective to use the barycenter of $\delta$ on the interval as the optimal estimation $\delta_{\text {opt }}$ rather than $\delta_{\max }$, which is sensitive to noises, i.e.,

$$
\delta_{\mathrm{opt}}=\int_{\delta_{\max }-\sigma_{\delta}}^{\delta_{\max }+\sigma_{\delta}} \delta \cdot f(\delta) d \delta
$$

\section{FIngERPRINT Matching}

The task of fingerprint matching is to obtain the minimal difference between input fingerprint $I$ and template $T$ by an optimal alignment. In this paper, however, deformation in fingerprints may bring false matching of local structures and therefore affects the final result. Thus, the global fingerprint matching is essential after the coarse local matching if the transformation model is known. In this process, the variable bounded box [9] is used to recheck all local matched structures to reduce the influence of deformation in fingerprints. The matching steps are listed as follows.

Step 1) For each comprehensive minutia pair $M_{i}$ and $M_{t}$, where $M_{i} \in M^{I}$ and $M_{t} \in M^{T}$, calculate the connected subgraph similarity $\hat{S}_{i t}$, formally

$$
\hat{S}_{i t}=\sum_{n \in R^{I}(n)} \sum_{m \in R^{T}(m)} S_{n m}
$$

where $R^{I}(n)=\left\{k \mid E_{k} \in E^{I}, s_{k}=n\right.$, or $\left.e_{k}=n\right\}$, and $R^{T}(m)=\left\{k \mid E_{k} \in E^{T}, s_{k}=m\right.$, or $\left.e_{k}=m\right\}$. $\hat{S}_{i t}$ is the similarity between the starlike subgraph centered at $M_{i}$ in $G^{I}$ and the starlike subgraph centered at $M_{t}$ in $G^{T}$.

Step 2) Set the iterative number $c=1$.

Step 3) Find the $c$ th maximum connected subgraph similarity $\hat{S}_{c i c t}$ and define the corresponding minutia pair $M_{c i}$ and $M_{c t}$ as the reference minutia pair.
Step 4) Use the reference minutiae $M_{c i}$ and $M_{c t}$ as the original points of the two graphs $G^{I}$ and $G^{T}$, respectively. All minutiae are aligned into their new polar systems and rotated with the statistical parameter $\delta_{\text {opt }}$.

Step 5) For each pair of matched local structures $E_{i}$ and $E_{t}$, where $E_{i} \in E^{I}, E_{t} \in E^{T}$, and $S_{i t} \neq 0$, if the two minutiae of $E_{i}$ are located within the variable bounded boxes [9] centered at the two minutiae of $E_{t}$, respectively, then $E_{i}$ and $E_{t}$ are true match; otherwise, they are false match.

Step 6) Calculate the similarities of two fingerprints $I$ and $T$ as follows:

$$
\begin{aligned}
\widetilde{S}_{c}=f_{\mathrm{GLM}}\left(n_{c} ; n t h 1, n t h 2\right) \\
\cdot f_{\mathrm{GLM}}\left(m_{c} ; m t h 1, m t h 2\right)
\end{aligned}
$$

where $n_{c}$ and $m_{c}$ denote the number and the similarity mean of true-matching local structures, respectively; nth 1 , nth 2 , $m t h 1$, and $m t h 2$ are four empirical values; and $f_{\mathrm{GLM}}(x ; t h 1, t h 2)$ is borrowed from the nonlinear matching technique, formally

$$
f_{\mathrm{GLM}}(x ; t h 1, t h 2)=\left\{\begin{array}{cc}
0, & x<t h 2 \\
\frac{x-t h 2}{t h 1-t h 2}, & t h 2 \leq x<t h 1 \\
1, & t h 1 \leq x .
\end{array}\right.
$$

Step 7) If $c<C$, go back to Step 3), where $C$ is the maximum iterative number and $C>1$.

Step 8) $\max _{1 \leq c<C}\left\{\tilde{S}_{c}\right\}$ is the optimal matching value of fingerprints $I$ and $T$; the larger the value is, the more similar the two fingerprints are. If the optimal matching value is more than the threshold $S t h$, the two fingerprints are considered from the same finger.

In our method, the thresholds, i.e., $\varepsilon$ and $S t h$, are estimated with the iterated conditional mode, which selects threshold by maximum entropy criterion [16]. The other empirical values, such as $C, L, d l$, and $d h$, are predetermined with many experiments on a training set.

\section{EXPERIMENTS}

We evaluate our algorithm on the fingerprint databases provided by FVC in 2002 [17], which are appropriate for testing online fingerprint systems. Our experiments analyzed the character of adaptive Parzen window, checked the validity of rotation parameter estimation, and evaluated the final matching performance.

\section{A. Character of the Probability Density Curve}

In this section, an experiment is performed to analyze the character of the probability density curve estimated by the adaptive Parzen window. The curve from the same fingerprint pair has been compared with that from different fingerprint pairs.

Three probability density curves are estimated, as shown in Fig. 2. The first curve is calculated with images A and B, which 


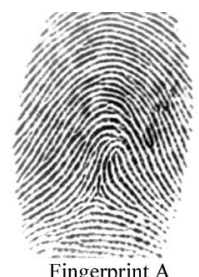

(1 1, DB1 a, FVC2002)
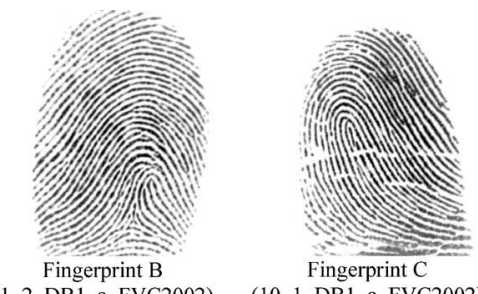

Fingerprint C

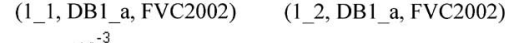

(10 1, DB1 a, FVC2002)
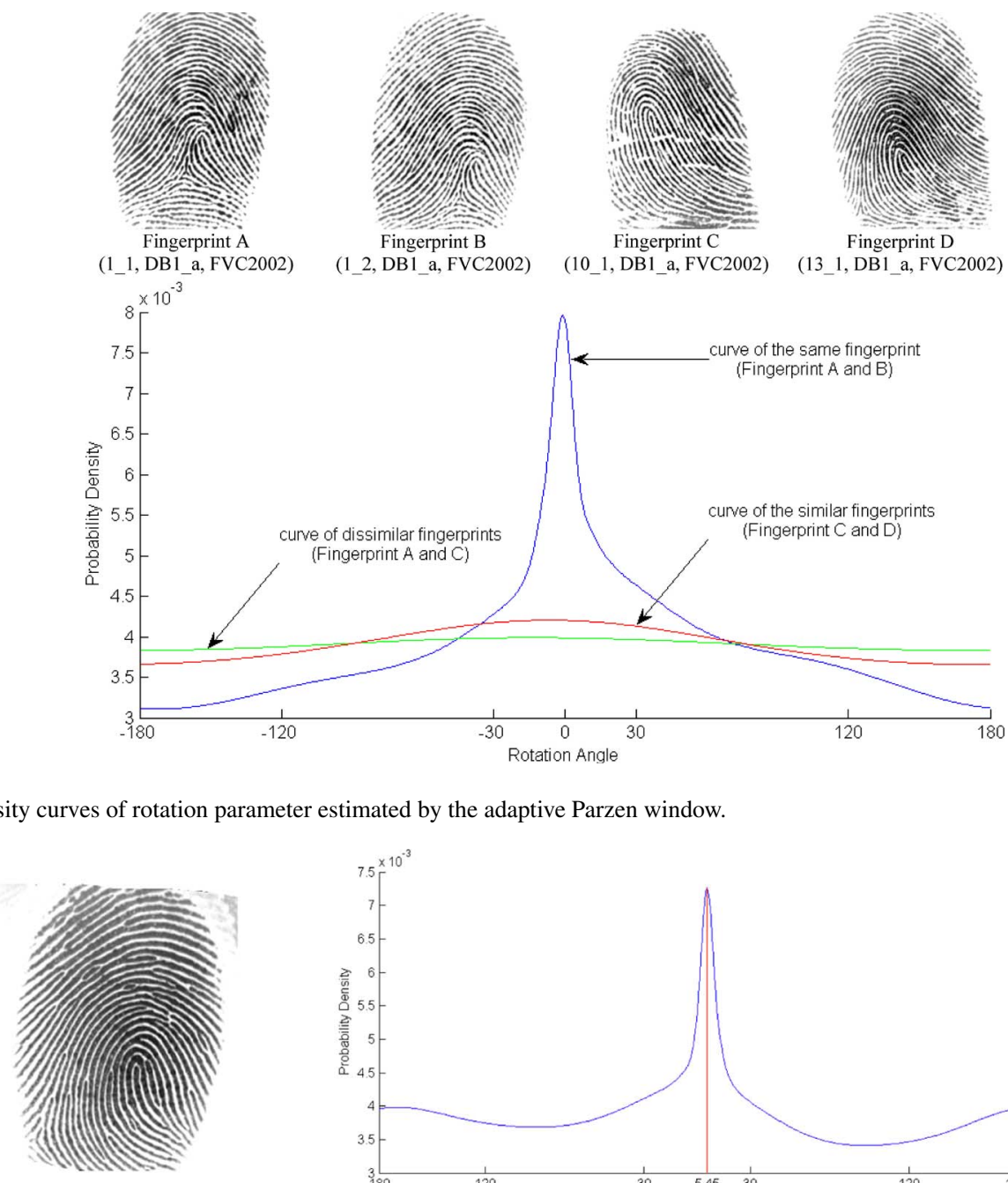
(Fingerprint $\mathrm{A}$ and $\mathrm{B}$ ) curve of dissimilar fingerprints
(Fingerprint $\mathrm{A}$ and $\mathrm{C}$ )

Fig. 2. Probability density curves of rotation parameter estimated by the adaptive Parzen window.
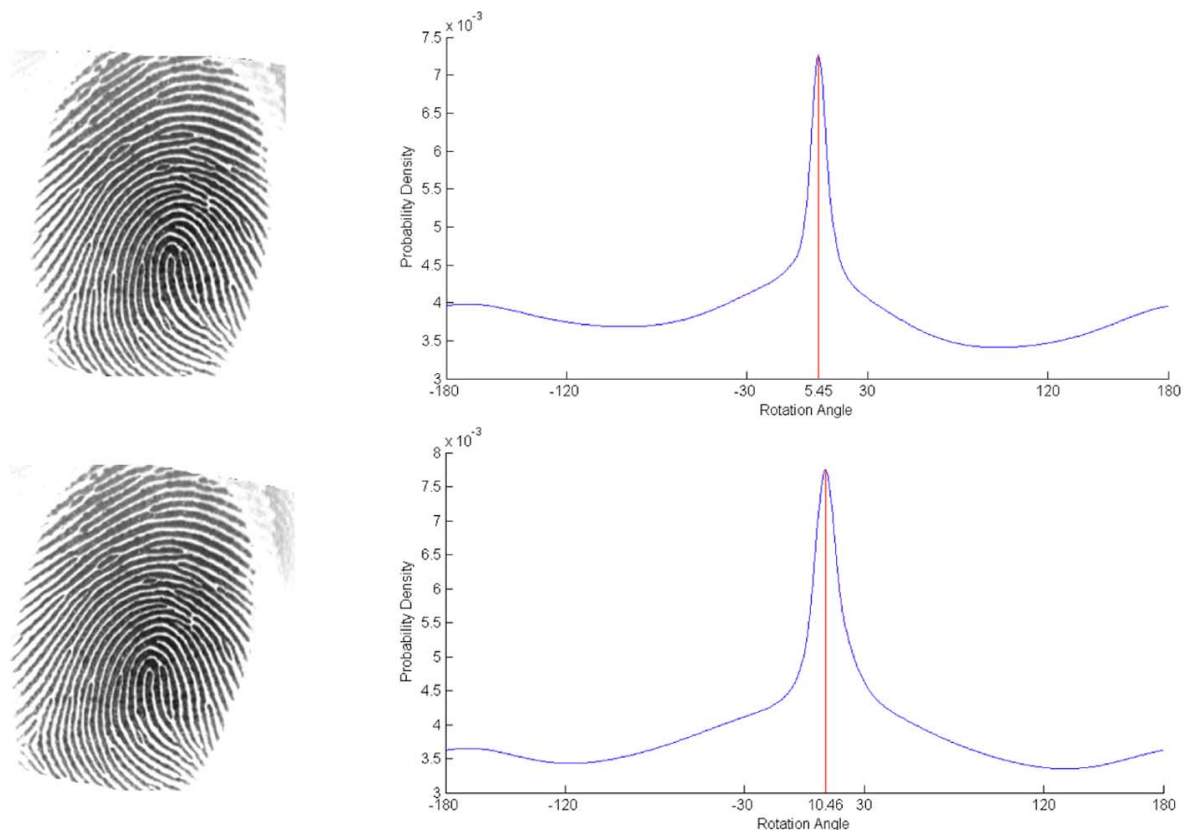

(1)

(2)
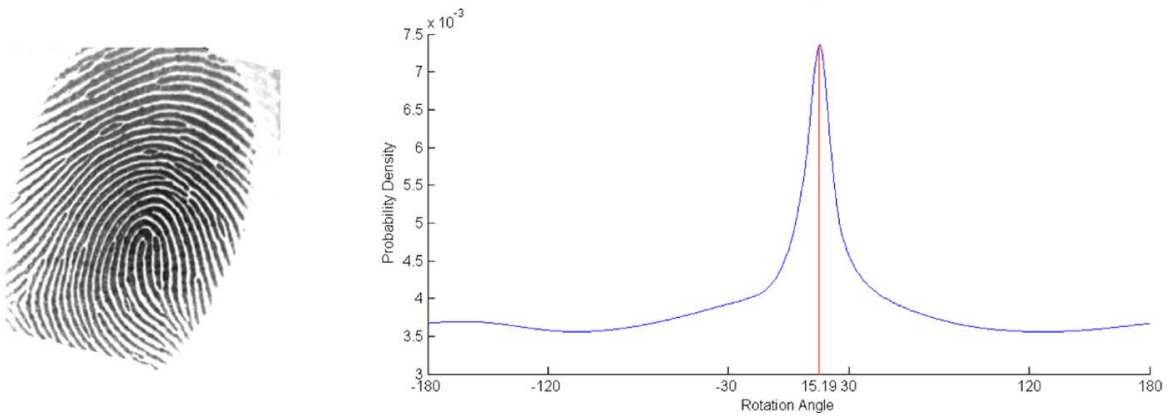

(3)

Fig. 3. Probability density curves under different global rotation parameters.

are acquired from the same finger. The second is estimated with images $\mathrm{C}$ and $\mathrm{D}$, which come from two similar fingers. The third is computed with images $\mathrm{A}$ and $\mathrm{C}$, which come from two dissimilar fingers. Among these three experiments, the curve of the same fingerprints is very sharp because there are many true-match local structure pairs, which contribute to 
TABLE I

ADAPTIVE PARZEN WINDOW VERSUS PARZEN WINDOW AND HISTOGRAM

\begin{tabular}{cccc}
\hline Real parameter & $\begin{array}{c}\text { Adaptive Parzen- } \\
\text { window Estimation }\end{array}$ & $\begin{array}{c}\text { Parzen-window } \\
\text { Estimation }\end{array}$ & $\begin{array}{c}\text { Histogram } \\
\text { Estimation }\end{array}$ \\
\hline 5.0000 & 5.4498 & 5.8008 & 5.6250 \\
10.0000 & 10.4590 & 10.7227 & 11.2500 \\
15.0000 & 15.1898 & 15.6445 & 14.0625 \\
$\begin{array}{c}\text { Average of } \\
\text { Absolute Errors }\end{array}$ & 0.3662 & 0.7227 & 0.9375 \\
\hline
\end{tabular}

the density around the true rotation angle. The curve of similar fingerprints is more convex than that of dissimilar fingerprints since several local structure pairs with the same rotation angle are accidentally matched. The experiment shows that the more similar the two fingerprints are, the sharper their probability density curve is. It is meaningful for quick rejection of impostor in practical recognition application. A simple way is to use the curve's peak value as the match value of two fingerprints, and if the value is under a threshold, the two fingerprints are considered from different fingers.

\section{B. Performance of the Adaptive Parzen Window}

Two experiments are conducted to evaluate the accuracy of the rotation parameter estimation with the adaptive Parzen window because the parameter plays a very important role in the fingerprint alignment and final matching.

In the first experiment, a fingerprint is selected randomly from FVC2002 database as the template $T$. Then, the fingerprint image is transformed with the angles $5^{\circ}, 10^{\circ}$, and $15^{\circ}$, respectively, as the input fingerprint $I$. Finally, the rotation parameter $\delta_{k}$ between $T$ and $I$ is estimated with the adaptive Parzen window, as illuminated in Fig. 3.

In the experiment, the adaptive Parzen window method is compared with the original method and the histogram method. As shown in Table I, the average error of our algorithm is below half of the Parzen window, and it is about one-third of the histogram estimation.

In the second experiment, a randomly selected fingerprint is rotated from $14^{\circ}$ to $-14^{\circ}$, and in total, 29 estimations are conducted, as illuminated in Table II and Fig. 4. The mean and standard deviation of the absolute errors are 0.420 and 0.242 , respectively.

\section{Matching Results on FVC2002}

To evaluate the overall matching performance of our method, a series of experiments are conducted over the four fingerprint databases of FVC2002. To judge whether the binary comprehensive minutia structure is helpful, the method without the binary structure [9], i.e., $\mathrm{Alg}_{-} 1$, is compared with the proposed method, named Alg_2. The receiving operating curves (ROCs) [18] illustrate the overall performance, as shown in Fig. 5.

Among the four data sets, the fingerprint quality of DB2_a is the best, whereas that of DB3_a is the worst [17]. As indicated by the ROCs, the proposed method outperforms the algorithm that does not involve the binary comprehensive minutia structures on FVC2002 databases. The equal error rates (EERs) of our method are $1.6 \%, 0.9 \%, 3.4 \%$, and 1.8\% in DB1_a, DB2_a,
TABLE II

Estimated Parameter $\delta_{k}$ Versus Real Parameter $K$

\begin{tabular}{ccc|ccc}
\hline \multicolumn{3}{c|}{ Clockwise } & \multicolumn{3}{c}{ Anticlockwise } \\
\hline$K$ & $\delta_{k}$ & Error & $K$ & $\delta_{k}$ & Error \\
\hline 0.0000 & 0.6666 & -0.6666 & 0.0000 & -0.6666 & 0.6666 \\
1.0000 & 0.7947 & 0.2053 & -1.0000 & -0.6447 & -0.3553 \\
2.0000 & 2.1465 & -0.1465 & -2.0000 & -1.9467 & -0.0533 \\
3.0000 & 3.1748 & -0.1748 & -3.0000 & -2.8743 & -0.1257 \\
4.0000 & 3.3572 & 0.6428 & -4.0000 & -3.1542 & -0.8458 \\
5.0000 & 4.7316 & 0.2684 & -5.0000 & -4.9378 & -0.0622 \\
6.0000 & 6.0095 & -0.0095 & -6.0000 & -6.1997 & 0.1997 \\
7.0000 & 7.1332 & -0.1332 & -7.0000 & -7.3364 & 0.3364 \\
8.0000 & 7.4404 & 0.5596 & -8.0000 & -7.5479 & -0.4521 \\
9.0000 & 8.5917 & 0.4083 & -9.0000 & -8.1973 & -0.8027 \\
10.0000 & 9.7634 & 0.2366 & -10.0000 & -9.5637 & -0.4363 \\
11.0000 & 10.0817 & 0.9183 & -11.0000 & -10.5413 & -0.4587 \\
12.0000 & 11.1290 & 0.8710 & -12.0000 & -11.3211 & -0.6789 \\
13.0000 & 12.4436 & 0.5564 & -13.0000 & -12.4537 & -0.5463 \\
14.0000 & 13.5593 & 0.4407 & -14.0000 & -13.6584 & -0.3416 \\
\hline \multicolumn{3}{c}{ Average of } & 0.4159 & \multicolumn{3}{c}{ Average of } & 0.4241 \\
Absolute Errors & \multicolumn{4}{c}{} \\
\hline
\end{tabular}

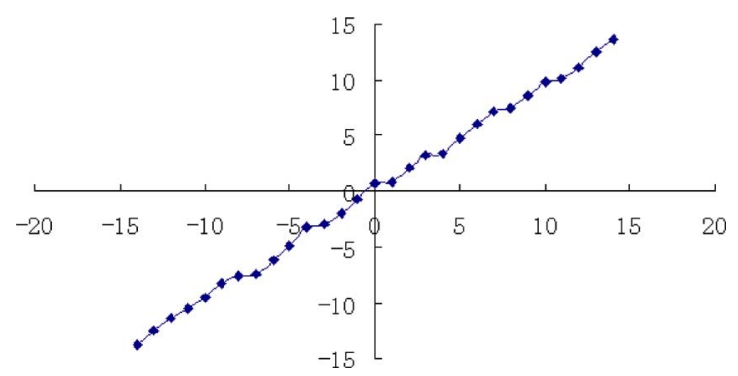

Fig. 4. Trend of the precision of estimated rotation angles.

DB3_a, and DB4_a, respectively, and the results are better than the best academic participants, i.e., PA24 and PA21, in FVC2002 [17]. With Pentium-III 933-MHz central processing unit, the average matching times are $0.37,0.55,0.27$, and $0.29 \mathrm{~s}$ in the four databases, respectively, which are much faster than the best industry participants, i.e., PA15 and PA27, in FVC2002 [17]. The better performance is contributed by two aspects: 1) minutia are replaced by binary minutia structure, and the structure has many effective features to represent a fingerprint; 2) the rotation parameter is accurately measured with the adaptive Parzen Window, and it makes satisfactory fingerprint alignment.

Additional experiment is conducted to compare the performance of binary minutia structure and ternary minutia structure in DB1_a and DB4_a of FVC2002. As illuminated in Table III, the EER of Chen's algorithm [19] with ternary minutia structure is about two-thirds of the proposed method, but in terms of the resource consumption, the proposed method is very competitive. By employing the binary minutia structure, our method makes a proper tradeoff between the performance and computational expense. This is important for applications that have limited computational resources.

\section{CONCLUSiOn AND FutURE WORK}

This paper introduces a robust fingerprint matching method based on the comprehensive features of fingerprint, and it employs two novel technologies: 1) the binary comprehensive minutia structure with the transformation-variant and 

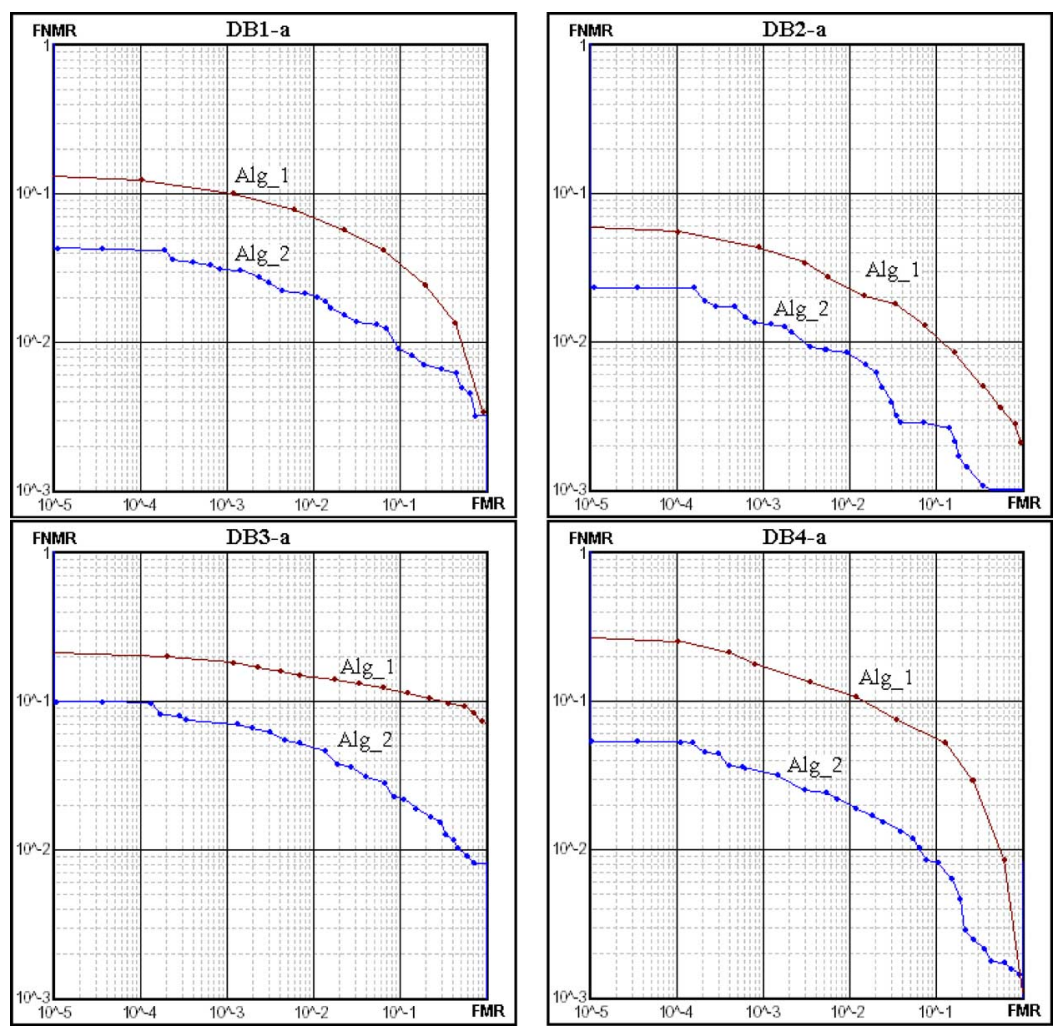

Fig. 5. ROCs of the matching experimental results over the four fingerprint databases of FVC2002.

TABLE III

BinARY STRUCTURE VERSUS TERNARY STRUCTURE. MMC Denotes MAXimum MeMORY CONSUMPTION, AND ATS STANDS FOR AVERAge TEMPLATE Size

\begin{tabular}{ccc}
\hline & $\begin{array}{c}\text { The proposed method } \\
\text { (binary structure) }\end{array}$ & $\begin{array}{c}\text { Chen's method } \\
\text { (ternary structure) }\end{array}$ \\
\hline EER & $1.7 \%$ & $1.3 \%$ \\
MMC & $4.0 \mathrm{Mb}$ & $12.5 \mathrm{Mb}$ \\
ATS & $1.3 \mathrm{~Kb}$ & $3.0 \mathrm{~Kb}$ \\
AMT & $0.33 \mathrm{~s}$ & $0.53 \mathrm{~s}$ \\
\hline
\end{tabular}

transformation-invariant features is implemented to provide a comprehensive representation of fingerprint. Meanwhile, it results in a graph representation of fingerprint. 2) The adaptive Parzen window is proposed to measure the transformation parameter. Compared with the traditional one, the adaptive Parzen window admits the periodic property, and it is more accurate by exploring the similarity information of local structure pairs. Moreover, it is more robust and needs fewer samples than the simple histogram estimation. By the way, the probability density curve, which is estimated by the adaptive Parzen window, shows the potential ability for fast impostor rejection.

Our method is based on the assumption that input fingerprint and the template are captured from the same sensor. In the case of different modal fingerprints [20], transformation invariants and variants of local structures will become invalid. Therefore, we will investigate the technique that employs a multiscale search strategy to address the issue. In addition, global pattern and features, as well as a hybrid matching technique, will be investigated to minimize false matching, which occasionally occurs with large deformation and very poor quality fingerprints.

\section{ACKNOWLEDGMENT}

The authors would like to thank Y. He for his grand contribution and crucial advice on this paper, as well as Y. Liu for revising the English.

\section{REFERENCES}

[1] A. Jain, L. Hong, S. Pankanti, and R. Bolle, "An identity authentication system using fingerprints," Proc. IEEE, vol. 85, no. 9, pp. 1365-1388, Sep. 1997.

[2] A. Jain, R. Bolle, and S. Pankanti, BIOMETRICS Personal Identification in Networked Society. Norwell, MA: Kluwer, 1999.

[3] X. Jiang and W. Yau, "Fingerprint minutiae matching based on the local and global structures," in Proc. 15th Int. Conf. Pattern Recog., 2000, vol. 2, pp. 1042-1045.

[4] X. Lou and J. Tian, "A minutia matching algorithm in fingerprint verification," in Proc. 15th Int. Conf. Pattern Recog., 2000, vol. 4, pp. 833-836.

[5] A. Jain, A. Ross, and S. Prabhakar, "Fingerprint matching using minutiae and texture features," in Proc. 4th Int. Conf. Image Process., Thessaloniki, Greece, 2001, pp. 282-285.

[6] S. Gold and A. Rangarajan, "A graduated assignment algorithm for graph matching," IEEE Trans. Pattern Anal. Mach. Intell., vol. 18, no. 4, pp. 377-388, Apr. 1996.

[7] A. Hrechak and J. McHugh, "Automated fingerprint recognition using structural matching," Pattern Recognit., vol. 23, no. 8, pp. 893-904, 1990.

[8] Z. Kovacs-Vajna, "A fingerprint verification system based on triangular matching and dynamic time warping," IEEE Trans. Pattern Anal. Mach. Intell., vol. 22, no. 11, pp. 1266-1276, Nov. 2000.

[9] Y. He, J. Tian, X. Luo, and T. Zhang, "Image enhancement and minutiae matching in fingerprint verification," Pattern Recognit. Lett., vol. 24, no. 9, pp. 1349-1360, Jun. 2003.

[10] D. Maio and D. Maltoni, "Direct gray-scale minutiae detection in fingerprints," IEEE Trans. Pattern Anal. Mach. Intell., vol. 19, no. 1, pp. 27-40, Jan. 1997.

[11] L. Hong and A. Jain, "Classification of fingerprint images," in Proc. 11th Scandinavian Conf. Image Anal., Kangerlussuaq, Greenland, 1999, pp. 781-795. 
[12] J. Chang and K. Fan, "A new model for fingerprint classification by ridge distribution sequences," Pattern Recognit., vol. 35, no. 6, pp. 1209-1223, Jun. 2002.

[13] A. Jain, S. Prabhakar, L. Hong, and S. Pankanti, "Filterbank-based fingerprint matching," IEEE Trans. Image Process., vol. 9, no. 5, pp. 846-859, May 2000

[14] M. Tico and P. Kuosmanen, "Fingerprint matching using an orientationbased minutia descriptor," IEEE Trans. Pattern Anal. Mach. Intell., vol. 25, no. 8, pp. 1009-1014, Aug. 2003.

[15] Y. He and J. Tian, "Fingerprint matching based on global comprehensive similarity," IEEE Trans. Pattern Anal. Mach. Intell., vol. 28, no. 6, pp. 850-862, Jun. 2006.

[16] X. Lou and J. Tian, "The ICM algorithm for multi-level threshold selection by maximum entropy criterion," J. Softw., vol. 11, no. 3, pp. 379-385, 2000. (in Chinese, with English abstract).

[17] D. Maio, D. Maltoni, R. Cappelli, J. Wayman, and A. Jain, "FVC2002: Second fingerprint verification competition," in Proc. 16th Int. Conf. Pattern Recog., 2002, vol. 3, pp. 811-814.

[18] D. Maio, D. Maltoni, R. Cappelli, J. Wayman, and A. Jain, "FVC2000: Fingerprint verification competition," IEEE Trans. Pattern Anal. Mach. Intell., vol. 24, no. 3, pp. 402-412, Mar. 2002.

[19] X. Chen, J. Tian, and X. Yang, "An novel algorithm for distorted fingerprint matching based on fuzzy features match," in Proc. 5th Int. Conf. Audio- and Video-Based Biometric Person Authentication, 2005, pp. 665-673.

[20] Y. He, J. Tian, R. Qun, and X. Yang, "Maximum-likelihood deformation analysis of different-sized fingerprints," in Proc. 4th Int. Conf. Audio- and Video-Based Biometric Person Authentication, 2003, pp. 421-428.

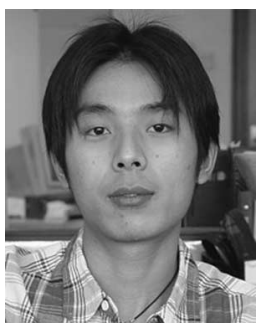

Xiaoguang He received the B.S. degree from the Special Class for the Gifted Young, University of Science and Technology of China, Beijing, China, in 2002 . He is currently working toward the Ph.D. degree at the Key Laboratory of Complex Systems and Intelligence Science, Institute of Automation, Chinese Academy of Sciences, Beijing.

His research interests include pattern recognition, machine learning, and image processing and their applications in biometrics.

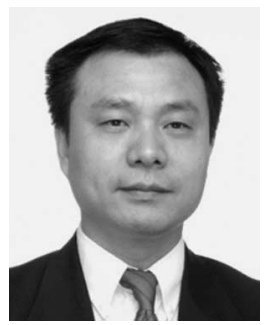

Jie Tian (M'01-SM'03) received the Ph.D. degree (with honors) in artificial intelligence from the Chinese Academy of Sciences (CAS), Beijing, China, in 1992.

From 1994 to 1996, he was a Postdoctoral Fellow in the Medical Image Processing Group, University of Pennsylvania, Philadelphia. Since 1997, he has been a Professor in the Key Laboratory of Complex Systems and Intelligence Science, Institute of Automation, CAS. He is also with the Life Science Center, Xidian University, Xi' an, China. He has published more than 50 papers in academic journals and international conferences. His research interests include medical image process and analysis and pattern recognition. He is a specially invited Reviewer for Mathematical Reviews.

Prof. Tian is the recipient of the National Scientific and Technological Progress Awards in 2003 and 2004.

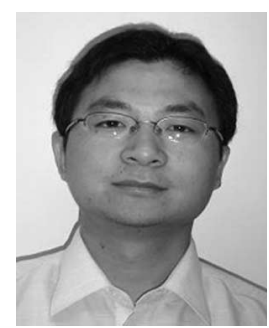

Liang $\mathbf{L i}$ received the B.S. degree from Northwestern Polytechnical University, Xi' an, China, in 2002. He is currently working toward the Ph.D. degree at the Key Laboratory of Complex Systems and Intelligence Science, Institute of Automation, Chinese Academy of Sciences, Beijing, China.

His research interests include pattern recognition, machine learning, and image processing and their applications in biometrics.

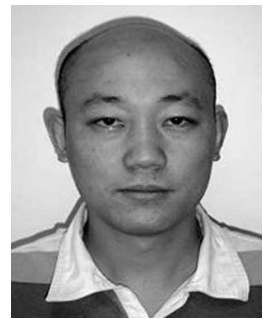

Yuliang He received the B.S. degree in computer and system science from Nankai University, Tianjin, China, in 1997 and the M.S. and Ph.D. degrees from the Institute of Automation, Chinese Academy of Sciences (CAS), Beijing, China, in 2003 and 2006, respectively.

$\mathrm{He}$ is currently with the Key Laboratory of Complex Systems and Intelligence Science, Institute of Automation, CAS. His research interests include pattern recognition, machine learning, and information fusion and their applications in biometrics.

Dr. He received the Chinese Academy of Sciences President Scholarship in 2005 .

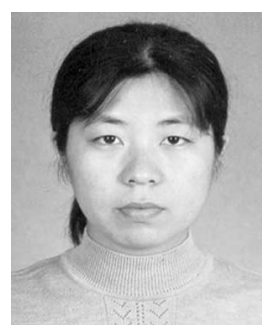

Xin Yang received the B.S., M.S., and Ph.D. degrees in intelligent instruments from Tianjin University, Tianjin, China, in 1994, 1997, and 2000, respectively.

From 2001 to 2003, she was a Postdoctoral Fellow with the Biometric Research Group, Key Laboratory of Complex Systems and Intelligence Science, Institute of Automation, Chinese Academy of Sciences, Beijing, China. Since 2003, she has been an Associate Professor with the laboratory. Her research interests include bioinformatics, pattern recognition, etc. 